InterSedes, Revista electrónica de las sedes regionales de la Universidad de Costa Rica, ISSN 2215-2458, Vol XXI, Número 43, Enero - Julio, 2020.

10.15517/isucr.v21i43.41972 | intersedes.ucr.ac.cr | intersedes@ucr.ac.cr

\title{
DETERMINACIÓN DEL TAMAÑO Y LA FORMA DE UNIDAD EXPERIMENTAL, CON EL MÉTODO DE REGRESIÓN MÚLTIPLE, PARA ENSAYOS DE RENDIMIENTO DE MAÍZ (ZEA MAYS), GUANACASTE, COSTA RICA
}

\section{SIZE AND SHAPE DETERMINATION OF AN EXPERIMENTAL UNIT, WITH THE MULTIPLE REGRESSION METHOD, FOR CORN (ZEA MAYS) YIELD TRIALS, GUANACASTE, COSTA RICA}

Jorge Claudio Vargas-Rojas ${ }^{1}$

Juan Ramón Navarro-Flores ${ }^{2}$

\begin{tabular}{|l|l}
\hline Recibido: 05.04 .19 & Aprobado: 30.04 .20
\end{tabular}

DOI: $10.15517 /$ isucr.v21i43.41972

\section{Resumen}

Se realizó un estudio con el objetivo de determinar el tamaño y la forma de unidad experimental para ensayos en arroz en el cantón de Santa Cruz, Guanacaste, Costa Rica. Se sembró un ensayo de uniformidad y los datos obtenidos fueron empleados para aplicar el método regresión múltiple. En este método, la variabilidad residual, medida como coeficiente de variación, correspondiente a los diferentes tamaños y formas de unidad experimental que se pueden simular se modela en función del largo y ancho mediante una regresión múltiple. Luego se estiman las derivadas parciales para cada regresora y se igualan a -1 ; al resolver este sistema de ecuaciones se obtiene la combinación de ancho y largo de unidad de experimental que minimiza el coeficiente de variación. Para este trabajo esa combinación resultó ser de 8 metros de largo por 4 metros de ancho para una unidad experimental de $32 \mathrm{~m}^{2}$.

Palabras clave: tamaño de unidad experimental; forma de unidad ex perimental; maíz; regresión múltiple; rendimiento.

\footnotetext{
Abstract

${ }^{1}$ Docente Sede Regional de Guanacaste, Universidad de Costa Rica, Liberia, Costa Rica.

Email: jorgeclaudio.vargas@ucr.ac.cr

${ }^{2}$ Docente. Centro de Investigación en Protección de Cultivos, Universidad de Costa Rica, San José, Costa Rica.

Email: juan.navarro@ucr.ac.cr
} 
A study was conducted to determine the size and shape of the experimental unit for rice yield trials in the canton of Santa Cruz, Guanacaste, Costa Rica. A uniformity trial was planted and the data obtained were used to apply multiple regression method. In this method, the residual variability, measured as a coefficient of variation, corresponding to the different sizes and forms of experimental unit that can be simulated, was modeled according to the length and width by a multiple regression. Then the partial derivatives for each regressor were estimated and equaled to -1 ; the result of this system of equations finds the combination of width and length of the experimental unit that minimizes the coefficient of variation. For this work the resulting combination was 8 meters long by 4 meters wide for an experimental unit of $32 \mathrm{~m}^{2}$.

Key words: plot size; plot shape; corn; multiple regression; yield.

\section{Introducción}

La unidad experimental (parcela) es el área en la que se lleva a cabo la asignación aleatoria de los tratamientos en una prueba crítica (Gomez 1972). Barros y Tavares (1995) la definen como la unidad básica que provee la información en la cual se basa la experimentación. Esta información, que se convertirá en los resultados del ensayo, proviene de pruebas de naturaleza probabilística y, por lo tanto, está ligada no solo al comportamiento que puede expresar un determinado tratamiento sino que a otros factores ajenos al mismo, que tienden a opacar su verdadero efecto, llamados en conjunto: error experimental (Barrientos - García, 1981).

Idealmente, las unidades experimentales deberían ser homogéneas de modo que luego de aplicarles distintos tratamientos se pueda decir que la diferencia (si existe) es exclusivamente producto del efecto de los tratamientos aplicados y no debido a otros factores, que en conjunto se denominan "ruido". No obstante, conseguir un conjunto de unidades experimentales totalmente homogéneas resulta imposible, particularmente, cuando se realizan ensayos en un campo agrícola, debido a la variabilidad espacial de las características fisicoquímicas del suelo (Stroup, 2002). La falta de homogeneidad genera un efecto considerable sobre la magnitud de la variabilidad de los datos no atribuible a causas conocidas (Box, Hunter, \& Hunter, 2005), esto es: el error experimental. La variabilidad entre unidades experimentales de un ensayo agrícola se conoce como la variabilidad natural de un sitio y si no se controla o se cuantifica podría distorsionar la estimación de las medias de los tratamientos y su comparación. Por lo tanto, si se desea que los resultados de un ensayo sean confiables se debe aplicar técnicas experimentales adecuadas, entre las que se recomienda utilizar un tamaño y una forma adecuados de unidad experimental (Párraga y Chacín 2000). 
La bibliografía especializada señala dos razones de primordial importancia para justificar la necesidad de estimar un tamaño y una forma óptimos de unidad experimental (Sánchez et al., 2006; Rodríguez, Sánchez, \& Pacheco, 1993). La primera es reducir el error experimental del ensayo; la segunda se basa en aspectos económicos y prácticos, como ahorro de semilla, espacio, recursos económicos y trabajo. Parcelas con un tamaño inferior al óptimo pueden dar resultados poco confiables, por otro lado parcelas grandes pueden incurrir en gastos innecesarios de tiempo y recursos (Gomez 1972). Así pues, la mayor eficiencia de un ensayo se fundamenta en un tamaño y una forma óptimos de unidad experimental (Vallejo y Mendoza 1992).

Para determinar el tamaño y la forma de parcela existen métodos estadísticos muy variados, la mayoría parten de un ensayo blanco o ensayo de uniformidad (Rodríguez et al.1993; Custódio, 2010; Santos, Haesbaert, Lúcio, Storck \& Cargnelutti, 2012). Si bien existen otros métodos, este es el más preciso, con el inconveniente de que requiere de una inversión considerable de tiempo y recursos (Álvarez, Soto, \& Gómez, 1986). El ensayo blanco es una parcela de extensión relativamente grande que es tratada en toda su superficie uniformemente en cuanto a fertilización, aplicación de agroquímicos y demás labores de cultivo y que a la hora de la cosecha se subdivide en parcelas pequeñas (unidades básicas) (Rosselló \& Fernández 1986), a partir de esta información existen diversos métodos para estimar el tamaño de unidad experimental.

Uno de los más utilizados, debido a su eficiencia, es el de regresión múltiple. En este las unidades básicas se agrupan de manera que formen parcelas de diferentes formas y tamaños, a estas parcelas resultantes se les cuantifica el rendimiento correspondiente mediante la adición de los rendimientos de las unidades básicas. Luego, para el total de unidades experimentales formadas con una combinación particular se estima la variabilidad residual, medida como coeficiente de variación, que quedará en función de la combinación del largo y del ancho de cada unidad experimental. Esto permite modelar el coeficiente de variación (CV) en función de largo y el ancho, lo que genera una superficie de respuesta en tres dimensiones, sobre la cual se puede estudiar los cambios en el CV debido a las variaciones del largo y del ancho y se puede obtener la combinación de estos que hagan que la superficie tenga una curvatura determinada. Usualmente, la curvatura que se busca es cuando la pendiente es -1; es decir, después de este punto añadir una nueva unidad básica en la variable independiente resultará en una reducción de la variable dependiente (CV) menor que la unidad, por lo que el incremento del tamaño ya no justifica la disminución en la variabilidad. Para minimizar 
la función se deben calcular las derivadas parciales del coeficiente de variación con respeto al largo y al ancho e igualar ambas a -1. Este método permite encontrar no solo el tamaño, sino también, la forma de parcela que minimice la variabilidad del ensayo (Álvarez et al. 1986; Barrientos - García, 1981).

El objetivo de este trabajo fue determinar el tamaño y la forma de unidad experimental para ensayos de rendimiento con arroz en la zona de Bagaces, Guanacaste, mediante el método de regresión múltiple.

Materiales y métodos

Generalidades

El ensayo se ejecutó durante los meses de junio a septiembre del año 2015, en la Finca Experimental de Santa Cruz, propiedad de la Universidad de Costa Rica, situada en el cantón de Santa Cruz, distrito de Santa Cruz, provincia de Guanacaste, Costa Rica. La misma se encuentra a 54 ms.n.m., posee una precipitación promedio de $1834 \mathrm{~mm} /$ año, temperatura media anual de 27,9 ${ }^{\circ}$ C. Para la siembra se utilizó semilla de maíz del híbrido HS5G. Esta se llevó a cabo de forma manual, en surcos separados a 1 metro y con una distancia entre plantas de $0,25 \mathrm{~m}$ para una densidad de 40000 plantas/ha. El manejo que recibió la parcela fue el mismo en todo lo relativo a fertilización, combate de arvenses, plagas y enfermedades.

Se empleó la técnica del ensayo blanco descrita por Rodríguez et al. (1993). De acuerdo con este método se sembró una parcela de maíz de $26 \mathrm{~m} \times 26 \mathrm{~m}$, es decir, de $676 \mathrm{~m}^{2}$. De la cual se dejó tres metros de borde alrededor de todo su perímetro; así se obtuvo un área de $20 \mathrm{~m} \times 20 \mathrm{~m}\left(400 \mathrm{~m}^{2}\right)$ para ejecutar el ensayo blanco.

Labores de campo

Cincuenta días después de la siembra se diseñó una cuadrícula sobre la parcela; para esto se empleó estacas de bambú y cuerdas, de modo que se identificarán claramente las 400 subparcelas (unidades 
básicas). Cada unidad básica correspondió a una hilera de un metro lineal. Además, cada una recibió coordenadas cartesianas que la ubicaran y la identificaran en el terreno; ambas coordenadas estuvieron dadas por distancias en metros a ejes cartesianos (X será el ancho y Y el largo de la parcela).

Se cosechó el elote entero de cada planta perteneciente a cada unidad básica y se colocaron en un saco previamente identificado con el número correspondiente según el sistema de coordenadas cartesianas. Se pesó cada uno de los sacos y se obtuvo la producción en gramos.

Análisis de datos

Con los datos ingresados (producción y coordenadas cartesiana de cada unidad básica) se conformó cada una de las posibles formas y tamaños de unidades experimentales que se obtuvieron por medio de la combinación de las unidades básicas y se calculó el coeficiente de variación (CV) respectivo. El CV se modeló en función del largo y el ancho de las unidades experimentales, según la Ecuación 1.

$$
C V_{i j k}=\beta_{0}+\beta_{1} \gamma_{i}+\beta_{2} \alpha_{j}+\beta_{3} \gamma_{i}^{2}+\beta_{4} \alpha_{j}^{2}+\beta_{5}(\gamma \alpha)_{i j}+\varepsilon_{i j k}
$$

donde:

$C V_{i j k}=k$-ésimo coeficiente de variación correspondiente al $i$-ésimo largo y al $j$-ésimo ancho.

$\gamma_{i}=i$-ésimo largo de parcela. Con $i: 1, \ldots, \mathrm{L}$.

$\alpha_{j}=j$-ésimo ancho de parcela. Con $i: 1, \ldots, \mathrm{A}$.

$(\gamma \alpha)_{i j}=$ interacción del $i$-ésimo largo con el $j$-ésimo ancho.

$\varepsilon_{i j k}=$ error aleatorio de la k-ésima observación del $i$-ésimo largo y $j$-ésimo ancho

Para cada uno de los términos del modelo de la Ecuación 1 se hizo la prueba de hipótesis respectiva para establecer si el valor estimado es distinto de cero o no. En caso de que alguno no fuera significativo ( $\mathrm{p}>0,05)$, este se excluyó del modelo. Todos los procedimientos se hicieron con el lenguaje R (R Core Team, 2017). 
InterSedes, $\mathbf{N}^{\circ}$ 43. Vol XXI (2020). ISSN 2215-2458

Resultados

En la segunda columna del Cuadro 1 se presenta las estimaciones de cada uno de los términos del modelo y en la quinta columna se encuentra el valor de probabilidad asociado a cada término. Se observa que todos los términos son significativos $(\mathrm{p}<0,001)$.

Cuadro 1. Coeficientes de regresión y estadísticos asociados para el modelo ajustado

\begin{tabular}{ccccc}
\hline Coeficiente & Estimación & Error estándar & Valor t & $\operatorname{Pr}(>|\mathrm{t}|)$ \\
\hline Ordenada & 39,84 & 2,18 & 18,23 & $<2 \mathrm{e}-16$ \\
Largo & $-2,72$ & 0,47 & $-7,35$ & $<0,001$ \\
Ancho & $-2,25$ & 0,45 & $-7,00$ & $<0,001$ \\
Largo $^{2}$ & 0,10 & 0,02 & 5,18 & $<0,001$ \\
Ancho $^{2}$ & 0,10 & 0,02 & 4,92 & $<0,001$ \\
Largo*Ancho $^{2}$ & 0,07 & 0,02 & 2,89 & $<0,001$ \\
\hline
\end{tabular}

El modelo estimado del coeficiente de variación en función del largo y del ancho se presenta en la Ecuación 2.

$$
C V=39,84-2,72 L-2,25 A+0,10 L^{2}+0,10 A^{2}+0,07 L * A
$$

Al estimar las derivadas parciales de la Ecuación 2 e igualares a -1, se obtuvo el siguiente sistema de ecuaciones:

$$
\left[\begin{array}{ll}
0,02 L & 0,07 A \\
0,07 L & 0,02 A
\end{array}\right]=\left[\begin{array}{l}
-1+2,25 \\
-1+2,72
\end{array}\right]
$$


Al resolver el sistema de la Ecuación 3 se obtuvo la combinación de largo y ancho en la cual se obtiene el punto de curvatura deseado. Para este caso esa combinación fue de 7,89 metros de largo y el ancho es de 3,60 metros; que para efectos prácticos se puede considerar como una unidad experimental de 8 metros de largo y 4 metros de ancho, o sea, 4 surcos de 8 metros de largo para un área de $32 \mathrm{~m}^{2}$.

\section{Discusión}

El tamaño que aquí se define corresponde al tamaño de parcela útil y no al de parcela total. Por lo que a este tamaño se le deben agregar los bordes que se consideren necesarios según sean las necesidades del ensayo.

El único trabajo encontrado en Costa Rica, sobre tamaño de unidad experimental, para el cultivo de maíz es el de Vargas y Navarro (2017), quienes en la misma zona de este trabajo establecieron un tamaño de $12 \mathrm{~m}^{2}$. En Venezuela, Monzón (1956) y Párraga y Chacín (2001) lo definieron en 12 $\mathrm{m}^{2}$ y $14 \mathrm{~m}^{2}$, respectivamente. En Colombia, Escobar (1981) recomendó una unidad experimental $20 \mathrm{~m}^{2}$. Mientras que Barrientos - García (1981), en Guatemala, estableció el tamaño óptimo en 20 $\mathrm{m}^{2}$. Todos los trabajos anteriores se basaron en el método de curvatura máxima para definir el tamaño de la unidad experimental. Barrientos - García (1981) también utilizaron el método de regresión múltiple y con este definió la unidad experimental como una combinación de 4 surcos (separados a un metro) de 9 metros de largo para un tamaño de $36 \mathrm{~m}^{2}$. Las variaciones, en menor o mayor medida, con los resultados encontrados en este trabajo puede deberse a que el tamaño de unidad experimental es una característica regional y está fuertemente afectada por las características agroecológicas de la zona donde se desarrolló el estudio (Chacín, 1977); además de que puede variar según el método de estimación empleado (Barrientos - García, 1981).

En comparación con el trabajo de Vargas y Navarro (2017), que fue desarrollado en la misma zona agroecológica, este trabajo propone un tamaño de unidad experimental de casi 3 veces al propuesto por estos autores. Si bien este trabajo establece un mayor tamaño es debido a que el método de regresión múltiple genera unidades experimentales más grandes en comparación con el método de curvatura máxima, tal y como lo encontró Barrientos - García (1981). Otro aspecto en lo que difiere este trabajo al presentado por Vargas y Navarro (2017) es en la forma, estos plantean una unidad experimental conformada por 2 surcos de 6 metros. Esta disposición, debido al método que 
utilizaron, proviene de una serie de otras disposiciones que fueron descartadas más por efectos prácticos que por reducción del CV. En este trabajo sí se presenta la combinación de largo y ancho que minimiza el CV; lo cual es una ventaja del método de regresión múltiple usado en este trabajo con respecto al método de curvatura máxima (Barrientos - García, 1981).

La heterogeneidad del suelo siempre va a estar presente en los ensayos realizados en el campo y es una de las principales causas del error experimental. Para minimizar este error, además de otras estrategias, se debe seleccionar el tamaño y la forma apropiados de la unidad experimental. Lamentablemente, a pesar de que el tamaño y la forma de unidad experimental se reporta como una herramienta valiosa para controlar el error experimental, su estudio ha perdido importancia en los últimos años, lo que se refleja en las escasas referencias bibliográficas de fechas recientes. Probablemente, debido al auge de tecnologías de cultivo intensivas donde grandes áreas son sometidas a los tratamientos. Sin embargo, en países pequeños, donde no existen estos sistemas de producción, donde hay limitantes de terreno y donde las labores de cultivo aún se realizan manualmente este tipo de investigación puede convertirse en una gran ayuda para los investigadores locales, en aras de maximizar los recursos y obtener resultados confiables.

\section{Bibliografía}

Álvarez, R., Soto, M., \& Gómez, H. (1986). Tamaño de parcela y número de repeticiones para estimar una población de Spodoptera frugiperda (Smith) y su daño en maíz. Revista Facultad Nacional de Agronomía, 39(2), 6-16.

Asif - Masood, M., Raza, I., \& Yaseen, M. (2012). Estimation of optimum field plot size and shape in paddy yield trial. Pakistan Journal of Agricultural Research, 25 (4), 280 - 287.

Barrientos García, M. (1981). Evaluación de 4 métodos para la determinación de tamaño y forma óptimos de parcela para experimentación agrícola. (Tesis inédita de licenciatura). Universidad de San Carlos, San Carlos, Guatemala. 79 p.

Barros, Inácio de, \& Tavares, Marcelo. (1995). Estimativa do tamanho ótimo de parcelas experimentais através de cálculos algébricos. Bragantia, 54(1), 209-215. https://dx.doi.org/10.1590/S0006-87051995000100024 
Box, G. E., Hunter, J. S., \& Hunter, W. G. (2005). Statistics for experimenters: Design, innovation and discovery (2nd ed.). Hoboken, NJ: Wiley-Interscience.

Chacín, F. 1977. Tamaño de parcela experimental y su forma. Revista de la Facultad de Agronomía de Maracay, 9(3), 55-74.

Custódio Silva da, W. (2010). Comparacao de métodos para estimar o tamaño ótimo de parcela embananeira. (Tesis inédita de maestría). Universidad Federal de Vicosa, Vicosa, Brasil. $37 \mathrm{p}$.

Gomez, K. A. (1972). Techniques for field experiments with rice: Layout, sampling, sources of error. Manila: International Rice Research Institute.

Gomez, K., \& Gomez, A. (1984). Statistical Procedures for Agricultural Research (2nd ed.). New York: Wiley.

Párraga, C., \& Chacín, F. (2000). Comparación de metodologías univariadas en la determinación de unidades experimentales de campo: renglón maíz (Zea mays L.). Revista Facultad de Agronomía de Maracay, 26(1), 175-190.

Rodríguez, N., Sánchez, H., \& Pacheco, P. (1993). Determinación de tamaño y forma óptimos de parcela para ensayos de rendimiento con café. Revista Colombiana de Estadística, 14(27), $50-64$.

Rosselló, J.M., \& Fernández, M. (1986). Guía técnica para ensayos de variedades de campo. FAO. $144 \mathrm{p}$.

Sánchez Hernández, M., Mejía Contreras, A., Villanueva Verduzco, C., Castellanos, J., Sánchez Hernández, C., \& Jiménez Rojas, C. (2006). Determinación del tamaño adecuado de parcela experimental en calabaza pipiana (Cucurbita argyrosperma hubervar. Stenosperma). Revista Fitotecnia Mexicana, 29(004), 339-348.

Santos, D., Haesbaert, F., Lúcio, A., Storck, L., \& Cargnelutti, A. (2012). Tamanho ótimo de parcela para a cultura do feijão-vagem. Revista Ciência Agronômica, 43(1), 119-128. https://dx.doi.org/10.1590/S1806-66902012000100015 
InterSedes, $\mathbf{N}^{\circ}$ 43. Vol XXI (2020). ISSN 2215-2458

Stroup, W. W. (2002). Power analysis based on spatial effects mixed models: A tool for comparing design and analysis strategies in the presence of spatial variability. Journal of Agricultural, Biological, and Environmental Statistics, 7(4), 491-511.

Vallejo, R., \& Mendoza, H. (1992). Plot technique studies on sweet potato yield trials. Journal of the American Society for Horticultural Science, 117(3), 508-511.

Vargas, J.C., \& Navarro, J.R. (2014). Determinación de un tamaño adecuado de unidad experimental, utilizando el método de curvatura máxima, para ensayos de arroz (Oryza sativa), en Bagaces, Guanacaste. Revista Intersedes, 15(31), 128-144. 